\title{
Cayley Graphs over LA-Groups and LA-Polygroups
}

\author{
Nabilah Abughazalah $\left(\mathbb{D},{ }^{1}\right.$ Naveed Yaqoob $\mathbb{D}^{2}$, and Asif Bashir ${ }^{2}$ \\ ${ }^{1}$ Mathematical Sciences Department, College of Science, Princess Nourah Bint Abdulrahman University, P.O. Box 84428, \\ Riyadh 11671, Saudi Arabia \\ ${ }^{2}$ Department of Mathematics and Statistics, Riphah International University, I-14, Islamabad, Pakistan
}

Correspondence should be addressed to Nabilah Abughazalah; nhabughazala@pnu.edu.sa

Received 6 April 2021; Accepted 27 April 2021; Published 10 May 2021

Academic Editor: Roslan Hasni

Copyright (C) 2021 Nabilah Abughazalah et al. This is an open access article distributed under the Creative Commons Attribution License, which permits unrestricted use, distribution, and reproduction in any medium, provided the original work is properly cited.

\begin{abstract}
The purpose of this paper is the study of simple graphs that are generalized Cayley graphs over LA-polygroups (GCLAP - graphs). In this regard, we construct two new extensions for building LA-polygroups. Then, we define Cayley graph over LA-group and GCLAP-graph. Further, we investigate a few properties of them to show that each simple graph of order three, four, and five (except cycle graph of order five which may or may not be a GCLAP-graph) is a GCLAP-graph and then we prove this result.
\end{abstract}

\section{Introduction}

The origins of graph theory can be traced back to Euler's work [1] on the königsberg bridges problem (1735), which thusly prompted the idea of an Eulerian graph. Graph is a mathematical portrayal of a grid and it portrays the relationship between lines and points.

The idea of Cayley graph was introduced by Cayley [2] in 1878. Cayley graph has been widely studied in both directed and undirected forms. To study the characteristics of Cayley graphs, refer the papers [3-6].

First time Marty [7] introduced the concept of algebraic hyperstructures, which is a suitable extension of classical algebraic structures. Since then, a lot of works have been written on this topic. For a brief analysis of this theory, see $[8,9]$. In the books [10-13], we can see the applications of hyperstructures in lattices, cryptography, graph, automata, probability, geometry, and hypergraphs. A very good presentation of polygroup theory is in [14], which is utilized to consider color algebra [15-17] and hypergraph theory in [18] by Berge.

The theory of left almost structures was first defined by Kazim and Naseeruddin [19] in 1972. Subsequently, Mushtaq and Kamran [20] established a new concept of left almost group (nonassociative group) called LA-group. The theory of left almost hyperstructures was first introduced by
Hila and Dine [21] in the form of left almost semihypergroups, which was further investigated by Yaqoob et al. [22] and Amjad et al. [23]. In [24], Yaqoob et al. introduced the concept of LA-polygroups.

Recently, Heidari et al. [25] introduced a suitable generalization of Cayley graphs that is defined over polygroups (GCP - graphs) and showed that each simple graph of order $\geq 5$ is a GCP-graph.

In this paper, we construct two new extensions for building LA-polygroups. Then, we define the idea of Cayley graph over LA-group and GCLAP-graph. In particular, we proved some properties of them in order to show that each simple graph of order three, four, and five (except cycle graph of order five which may or may not be a GCLAPgraph) is a GCLAP-graph.

\section{Preliminaries and Notations}

This section contains some basic definitions of graph theory (see [26]) and left almost theory (see [24]).

A graph is represented by $\Phi=(R, D)$, where $R$ is the set of vertices and $D$ is the set of edges. Note that $|R|$ is the order of a graph and $|D|$ in a graph is its size. The graph $K_{n}$ is known as complete graph if every couple of vertices form an edge, where $n$ is the number of vertices. In specific, $K_{1}$ is 
known as trivial graph and $N_{n}$ is known as null graph having no edges and $n$ vertices.

If $\Phi=(R, D)$ is a graph such that $R=R \prime \cup R^{\prime \prime}$ and $R^{\prime} \cap R^{\prime \prime}=\varnothing$, where $R^{\prime}$ and $R^{\prime \prime}$ are subsets of $R$ and edges of the form $\left\{\{d, f\} \mid d \in R^{\prime}\right.$ and $\left.f \in R^{\prime \prime}\right\}$, then $\Phi=K_{m, n}$ is known as complete bipartite graph, where $\left|R^{\prime}\right|=$ $m$ and $\left|R^{\prime \prime}\right|=n$. In specific, $K_{1, n}$ is known as star graph. The complement of a simple graph $\Phi=(R, D)$ is denoted by $\bar{\Phi}$, where $\bar{\Phi}=(R, \bar{D})$ such that $\bar{D}=\{\{d, f\} \mid\{d, f\} \notin D\}$. Let $\Phi=(R, D)$ and $\Phi^{\prime}=\left(R^{\prime}, D^{\prime}\right)$ be two graphs. Then, $\Phi^{\prime}$ is known as subgraph of $\Phi$, if $R^{\prime} \subseteq R$ and $D^{\prime} \subseteq D$. A subgraph $\Phi^{\prime} \subseteq \Phi$ is known as induced subgraph, if $\Phi^{\prime}$ contains each and every edge $\{d, f\} \in D$ with $d, f \in R^{\prime}$. Two graphs $\Phi=(R, D)$ and $\Phi^{\prime}=\left(R^{\prime}, D^{\prime}\right)$ are said to be isomorphic, if $\exists$, a bijection, $\quad \gamma: R \longrightarrow R^{\prime}$ such that $\{z, a\} \in D \Leftrightarrow$ $\{\gamma(z), \gamma(a)\} \in D^{\prime}$. We denote this by $\Phi \cong \Phi^{\prime}$. Let $\Phi=(R, D)$ and $\Phi^{\prime}=\left(R^{\prime}, D^{\prime}\right)$ be two graphs. Then, $\Phi \cup \Phi^{\prime}=\left(R^{\prime \prime}, D^{\prime \prime}\right)$, where $R^{\prime \prime}=R \cup R^{\prime}$ and $D^{\prime \prime}=D \cup D^{\prime}$ and joint of two graphs $\Phi \vee \Phi^{\prime}=\left(R^{\prime \prime}, D^{\prime \prime}\right)$ with $R^{\prime \prime}=R \cup R^{\prime}$ and $D^{\prime \prime}=D \cup D^{\prime} \cup\left\{\{d, f\} \mid d \in R, f \in R^{\prime}\right\}$. A graph $\Phi=(R, D)$ having no self edges and no multiple edges is known as simple graph.

Definition 1 (see [27]). If $\Phi=(R, D)$ is a graph and we form a sequence of vertices (ordered from left to right) $d_{1}, d_{2}, d_{3}, \ldots, d_{n}$ such that there is just one edge between every two succesive vertices and there are no other edges known as path. A path on $n$ vertices is denoted by $P_{n}$.

Definition 2 (see [27]). A graph is said to be a connected graph if there exists at least one path between every two vertices.

Definition 3 (see [27]). If all vertices have degree two of a connected graph, then it is called a cycle. A cycle graph has $n$ vertices, represented by $C_{n}$.

Definition 4 (see [20]). A groupoid $G$ is called a left almost group, i.e., LA-group, if

(i) $\exists e \in G$ such that $e d=d$ for all $d \in G$,

(ii) $\forall d \in G, \exists d^{*} \in G$ such that $d d^{*}=e$,

(iii) $(w f) d=(d f) w, \forall d, f, w \in G$.

Example 1 (see [20]). Let $G_{n}=\left\{t_{1}, t_{2}, \ldots, t_{n}\right\}$ where $n \geq 3$, under binary operation $*$ which is defined as

$$
t_{i} * t_{j}=t_{k}, \quad k \equiv(j+1)-i(\bmod n) .
$$

Then, $\left(G_{n}, *\right)$ is an LA-group. For $n=5$, we have the Cayley (Table 1).

Definition 5 (see [24]). A multivalued system $\left\langle L, \circ, e,^{-1}\right\rangle$, where $e \in L^{-1}$, is a unitary operation and $\circ$ maps $L \times L$ into the family of nonempty subsets of $L$ which is called LApolygroup, if the following postulates hold for all $d, f, w \in L$ :

(i) Left invertive law: $(d \circ f) \circ w=(w \circ f) \circ d$,

(ii) Reproducibility axiom: $d \circ L=L \circ d=L$,
TABLE 1: LA-group.

\begin{tabular}{llllll}
\hline$*$ & $t_{1}$ & $t_{2}$ & $t_{3}$ & $t_{4}$ & $t_{5}$ \\
\hline$t_{1}$ & $t_{1}$ & $t_{2}$ & $t_{3}$ & $t_{4}$ & $t_{5}$ \\
$t_{2}$ & $t_{5}$ & $t_{1}$ & $t_{2}$ & $t_{3}$ & $t_{4}$ \\
$t_{3}$ & $t_{4}$ & $t_{5}$ & $t_{1}$ & $t_{2}$ & $t_{3}$ \\
$t_{4}$ & $t_{3}$ & $t_{4}$ & $t_{5}$ & $t_{1}$ & $t_{2}$ \\
$t_{5}$ & $t_{2}$ & $t_{3}$ & $t_{4}$ & $t_{5}$ & $t_{1}$ \\
\hline
\end{tabular}

(iii) $\exists$ is a left identity $L \ni e$ such that $f=e \circ f$,

(iv) $f^{-1} \circ f \cap f \circ f^{-1} \sum e$,

(v) $d \in f \circ w \Longrightarrow f \in d \circ w^{-1}$.

Example 2 (see [24]). Consider a finite set $Q_{n}=$ $\left\{t_{1}, t_{2}, t_{3}, \ldots, t_{n}\right\}$, where $n \geq 3$. Then, $\mathbf{Q}_{n}$ is an LA-polygroup under the following hyperoperation:

$$
t_{i} * t_{j}= \begin{cases}t_{j}, & \text { for } i=1, \\ t_{k}, & \text { for } j=1 \text { and } k \equiv 2-i \bmod \left|Q_{n}\right|, \\ Q_{n}, & \text { for } j \neq 1, i \neq 1, i=j, \\ Q_{n} \backslash\left\{t_{1}\right\}, & \text { for } j \neq 1, i \neq 1, i \neq j .\end{cases}
$$

For $n=5$, we have the Cayley (Table 2).

Example 3 (see [24]). Consider $L_{1}=\{1,2,3,4\}$ with the hyperoperation $\circ$, given in Table 3 .

Then, $\mathbf{L}_{1}=\left\langle\{1,2,3,4\}, \circ, 1,{ }^{-1}\right\rangle$ is an LA-polygroup.

Notations. All LA-polygroups are represented by $\mathbf{L}, \mathbf{M}, \mathbf{N}, \ldots$ and the underlying sets are represented by $L, M, N, \ldots$ Also, $L^{*}=L \backslash\{e\}$ and $A^{-1}=\left\{a^{-1}: a \in A\right\}$. Now, we establish two new extensions for making LA-polygroups.

2.1. (I) Extension of an LA-Polygroup by a Set: $\mathbf{L}\{T\}$. Suppose that $\left\langle L, \circ, e,^{-1}\right\rangle$ is an LA-polygroup and $L \cap T=\varnothing$, where $T$ is a nonempty set. Put $M=L \cup T$, $e \uplus d=d$, for every $d \in M$ and $\forall d, f \in M^{*}=\{d \in M: d \neq e\}$; we define

$$
\begin{gathered}
d^{-I}= \begin{cases}d^{-1}, & \text { if } d \in L, \\
d, & \text { if } d \in T,\end{cases} \\
d \uplus e= \begin{cases}d \circ e, & \text { if } d \in L, \\
d, & \text { if } d \in T,\end{cases} \\
d \uplus f= \begin{cases}(d \circ f) \cup T, & \text { if } d, f \in L, \\
L \cup T, & \text { if } d=f \in T, \\
M^{*}, & \text { otherwise. }\end{cases}
\end{gathered}
$$

The system $\left\langle M, \uplus, e,^{-I}\right\rangle$ is known as the extension of LApolygroup $\mathbf{L}$ by a set $T$ and represented by $\mathbf{L}\{T\}$.

Theorem 1. Let $\left\langle L, \circ, e,^{-1}\right\rangle$ be an LA-polygroup and $T$ be a nonempty set such that $L \cap T=\varnothing$. Then, $\mathbf{L}\{T\}$ is an $L A-$ polygroup. 
TABLE 2: LA-polygroup.

\begin{tabular}{cccccc}
\hline$*$ & $t_{1}$ & $t_{2}$ & $t_{3}$ & $t_{4}$ & $t_{5}$ \\
\hline$t_{1}$ & $t_{1}$ & $t_{2}$ & $t_{3}$ & $t_{4}$ & $t_{5}$ \\
$t_{2}$ & $t_{5}$ & $Q_{n}$ & $Q_{n} \backslash\left\{t_{1}\right\}$ & $Q_{n} \backslash\left\{t_{1}\right\}$ & $Q_{n} \backslash\left\{t_{1}\right\}$ \\
$t_{3}$ & $t_{4}$ & $Q_{n} \backslash\left\{t_{1}\right\}$ & $Q_{n}$ & $Q_{n} \backslash\left\{t_{1}\right\}$ & $Q_{n} \backslash\left\{t_{1}\right\}$ \\
$t_{4}$ & $t_{3}$ & $Q_{n} \backslash\left\{t_{1}\right\}$ & $Q_{n} \backslash\left\{t_{1}\right\}$ & $Q_{n}$ & $Q_{n} \backslash\left\{t_{1}\right\}$ \\
$t_{5}$ & $t_{2}$ & $Q_{n} \backslash\left\{t_{1}\right\}$ & $Q_{n} \backslash\left\{t_{1}\right\}$ & $Q_{n} \backslash\left\{t_{1}\right\}$ & $Q_{n}$ \\
\hline
\end{tabular}

TABLE 3: LA-polygroup.

\begin{tabular}{ccccc}
\hline$\circ$ & 1 & 2 & 3 & 4 \\
\hline 1 & 1 & 2 & 3 & 4 \\
2 & 4 & $\{2,3,4\}$ & $\{2,4\}$ & $\{1,2,3\}$ \\
3 & 3 & $\{2,4\}$ & $\{1,3\}$ & $\{2,4\}$ \\
4 & 2 & $\{1,3,4\}$ & $\{2,4\}$ & $\{2,3,4\}$ \\
\hline
\end{tabular}

Proof. Suppose that $\mathbf{L}\{T\}=\mathbf{M}, L \cup T=M, L \cap T=\varnothing$, and $d, f, w \in M$. If $\{d, f, w\} \subseteq L^{*}$, then

$$
\begin{aligned}
(d \uplus f) \uplus w & =[(d \circ f) \circ w \cup T] \cup M^{*} \\
& =[(w \circ f) \circ d \cup T] \cup M^{*} \\
& =(w \biguplus f) \uplus d .
\end{aligned}
$$
then

If exactly one of $d, f, w \in L$ is equal to the left identity,

$$
(d \uplus f) \uplus w=(d \circ f) \circ w \cup T=(w \circ f) \circ d \cup T=(w \uplus f) \uplus d .
$$

If exactly two of $d, f, w \in L$ are equal to the left identity, then

$$
(d \uplus f) \uplus w=(d \circ f) \circ w=(w \circ f) \circ d=(w \uplus f) \biguplus d .
$$

If $\{d, f, w\} \nsubseteq L$, then $\quad(d \uplus f) \uplus w=M=(w \uplus f) \uplus d$. Thus, left invertive law holds. Now, we prove axiom $(v)$ of Definition 5. Let $d, f, w \in M$ such that $w \in d \uplus f$, then

Case 1. If $d, f, w \in L$, then we have done.
Case 2. (a) If $w \in L^{*}$, then we have the following possibilities:

(i) If $d \in T$ and $f \in L^{*}$, then $T \subseteq w \biguplus f^{-I} \Rightarrow d \in w \uplus f^{-I}$;

(ii) If $f \in T$ and $d \in L^{*}$, then $d \in w \uplus f^{-I}=w \uplus f=M^{*} \Rightarrow d \in w \biguplus f^{-I} ;$

(iii) If $d, f \in T$, then $d \in w \uplus f^{-I}=w \uplus f=M^{*} \Rightarrow d \in w \uplus f^{-I}$.

Case 2. (b) If $w=e$, then $w \in d \uplus f \Rightarrow d=f^{-I}$ and $w \uplus f^{-I}=e \uplus d=\{d\} \Rightarrow d \in w \uplus f^{-I}$.

Case 3. (a) If $w \notin L$ and $d, f \in M^{*}$, then

$$
\begin{cases}w \uplus f^{-I}=M^{*}, & \text { if } w \neq f, \\ w \uplus f^{-I}=M, & \text { if } w=f .\end{cases}
$$

Hence, $d \in w \uplus f^{-I}$.

Case 3. (b) If $w \notin L$ and $d=e$, then $w \in d \uplus f \Rightarrow w=$ $f \in T$ and $w \uplus f^{-I}=f \uplus f^{-I}=f \uplus f=M$, hence $d \in w \uplus f^{-I}$.

Case 3. (c) If $w \notin L$ and $f=e$, then

$w \in d \uplus f=\{d\} \Rightarrow w=d$ and $w \uplus f^{-I}=\{w\}=\{d\}$, hence $d \in w \biguplus f^{-I}$.

Thus, condition $(v)$ of Definition 5 holds and hence the theorem is proved.

Example 4. Let $\mathbf{L}_{2}=\left\langle\{1,2,3,4\}, \circ, 1,{ }^{-1}\right\rangle$ be an LA-polygroup with the Cayley (Table 4 ).

Then, $\mathbf{M}_{2}=\mathbf{L}_{2}\{\{5,6\}$ is an LA-polygroup with six elements and the Cayley (Table 5), where $M_{2}=\{1,2,3,4,5,6\}$.

2.2. (II) Extension of an LA-Polygroup by a Set: $\mathbf{L}\{T\}^{\diamond}$. Suppose that $\left\langle L, \circ, e,{ }^{-1}\right\rangle$ is an LA-polygroup and $L \cap T=\varnothing$, where $T$ is a nonempty set such that $T=\cup_{i=1}^{n} t_{i}$ (ordered from left to right, i.e., $\left.t_{1}, t_{2}, t_{3}, \ldots, t_{n}\right)$. Put $L \cup T=M$ and $e \uplus d=d, \forall d \in M$. We define

$$
\begin{aligned}
& d^{-I}= \begin{cases}d^{-1}, & \text { if } d \in L, \\
d, & \text { if } d \in T,\end{cases} \\
& d \uplus e= \begin{cases}d \circ e, & \text { if } d \in L, \\
d, & \text { if } d \in T,\end{cases} \\
& d \uplus f= \begin{cases}d \circ f, & \text { if } d, f \in L, \\
t_{i}, & \text { if } d \in L, f=t_{i} \in T \text { OR } d=t_{i} \in T, f \in L \text { where } 1 \leq i \leq n, \\
t_{i}, & \text { if } d=t_{i}, f=t_{j} \in T \text { where } i>j, \\
t_{j}, & \text { if } d=t_{i}, f=t_{j} \in T \text { where } i<j, \\
L, & \text { if } d=t_{1}=f \in T, \\
L \cup A_{i}, & \text { if } d=t_{i+1}=f \in T \text { where } 1 \leq i \leq n-1,\end{cases}
\end{aligned}
$$


TABLE 4: LA-polygroup.

\begin{tabular}{ccccc}
\hline$\circ$ & 1 & 2 & 3 & 4 \\
\hline 1 & 1 & 2 & 3 & 4 \\
2 & 3 & $\{2,3\}$ & $\{1,2\}$ & 4 \\
3 & 2 & $\{1,3\}$ & $\{2,3\}$ & 4 \\
4 & 4 & 4 & 4 & $\{1,2,3\}$ \\
\hline
\end{tabular}

TABLE 5: LA-polygroup.

\begin{tabular}{ccccccc}
\hline$\uplus$ & 1 & 2 & 3 & 4 & 5 & 6 \\
\hline 1 & 1 & 2 & 3 & 4 & 5 & 6 \\
2 & 3 & $\{2,3,5,6\}$ & $\{1,2,5,6\}$ & $\{4,5,6\}$ & $M_{2}^{*}$ & $M_{2}^{*}$ \\
3 & 2 & $\{1,3,5,6\}$ & $\{2,3,5,6\}$ & $\{4,5,6\}$ & $M_{2}^{*}$ & $M_{2}^{*}$ \\
4 & 4 & $\{4,5,6\}$ & $\{4,5,6\}$ & $\{1,2,3,5,6\}$ & $M_{2}^{*}$ & $M_{2}^{*}$ \\
5 & 5 & $M_{2}^{*}$ & $M_{2}^{*}$ & $M_{2}^{*}$ & $M_{2}$ & $M_{2}^{*}$ \\
6 & 6 & $M_{2}^{*}$ & $M_{2}^{*}$ & $M_{2}^{*}$ & $M_{2}^{*}$ & $M_{2}$ \\
\hline
\end{tabular}

Note that $M_{2}^{*}=M_{2} \backslash\{1\}=\{2,3,4,5,6\}$.

where $A_{i}=\cup_{h=1}^{i}\left\{t_{h}\right\}$. The system $\left\langle M, \uplus, e,^{-I}\right\rangle$ is known as the extension of LA-polygroup $\mathbf{L}$ by a set $T$ and represented by $\mathbf{L}\{T\}^{\diamond}$.

Theorem 2. Let $\left\langle L, \circ, e,^{-1}\right\rangle$ be an LA-polygroup and $T$ be a nonempty set such that $L \cap T=\varnothing$. Then, $\mathbf{L}\{T\}^{\diamond}$ is an $L A$ polygroup.
Proof. Suppose that $\mathbf{L}\{T\}^{\diamond}, L \cup T=M, L \cap T=\varnothing$, and $d, f, w \in M$.

If $\{d, f, w\} \subseteq L$, then clearly left invertive law holds. If $\{d, f, w\} \nsubseteq L$, then we consider the following cases:

Case 1. If $w \notin L$ and $d, f \in L$, then $(w \uplus f) \uplus d$ $=w=(d \uplus f) \uplus w$.

Case 2. If $w=s_{k}, f=s_{j} \notin L$, and $d \in L$, then

$$
\begin{array}{ll}
(d \uplus f) \uplus w=w \uplus w=(w \uplus f) \uplus d, & \text { if } k=j, \\
(d \uplus f) \uplus w=w=(w \uplus f) \uplus d, & \text { if } k>j, \\
(d \uplus f) \uplus w=f=(w \uplus f) \uplus d, & \text { if } k<j,
\end{array}
$$

Case 3. If $w=s_{k}, f=s_{j}, d=s_{i} \notin L$ in the following way:

(i) such that $k>j>i$, then

$$
\begin{aligned}
(d \uplus f) \uplus w & =\left(s_{i} \uplus s_{j}\right) \uplus s_{k}=s_{k} \\
& =\left(s_{k} \uplus s_{j}\right) \uplus s_{i}=(w \uplus f) \uplus d,
\end{aligned}
$$

(ii) such that $i=j$, then

$$
\begin{array}{ll}
(d \uplus f) \uplus w=\left(s_{i} \uplus s_{j}\right) \uplus s_{k}=s_{i} \uplus s_{i}=\left(s_{k} \uplus s_{j}\right) \uplus s_{i}=(w \uplus f) \uplus d, & \text { if } k<i=j \neq 1, \\
(d \uplus f) \uplus w=\left(s_{i} \uplus s_{j}\right) \uplus s_{k}=s_{k}=\left(s_{k} \uplus s_{j}\right) \uplus s_{i}=(w \uplus f) \uplus d, & \text { if } k>i=j,
\end{array}
$$

(iii) such that $j=k$, then

$$
\begin{array}{ll}
(d \uplus f) \uplus w=\left(s_{i} \uplus s_{j}\right) \uplus s_{k}=s_{j} \uplus s_{j}=\left(s_{k} \uplus s_{j}\right) \uplus s_{i}=(w \uplus f) \uplus d, & \text { if } i<j=k \neq 1, \\
(d \uplus f) \uplus w=\left(s_{i} \uplus s_{j}\right) \uplus s_{k}=s_{i}=\left(s_{k} \uplus s_{j}\right) \uplus s_{i}=(w \uplus f) \uplus d, & \text { if } i>j=k .
\end{array}
$$

Thus, left invertive law holds. Now, we prove axiom $(v)$ of Definition 5. Let $d, f, w \in M$ such that $w \in d \uplus f$. Then, we consider the following cases:

Case 1. If $w \in T$, then either $w=d \in T$ or $w=f \in T$ or $s_{1} \neq d=f \in T$ :

(i) If $w=d \in T$, then $d \in w \uplus f^{-I}=d \uplus f^{-I}=\{d\}$,

(ii) If $w=f \in T$ and $d \in L$, then $L \subseteq f \uplus f=w \uplus f^{-I}$ $\Rightarrow d \in w \uplus f^{-I}$; if $w=f \in T$ and $d \in T$, then $w=$ $f=s_{j}(j \neq 1)$ and $L \cup A_{j-1} \subseteq w \uplus f^{-I} \Rightarrow d \in w \uplus f^{-I}$,

(iii) If $s_{1} \neq d=f \in T$, then $w \uplus f^{-I}=w \uplus f=$ $f=d \Rightarrow d \in w \uplus f^{-I}$.

Case 2. If $w \in L$, then $d=f \in T$ and $w \uplus f^{-I}=w \uplus f$ $=f=d \Rightarrow d \in w \uplus f^{-I}$.
Thus, condition $(v)$ of Definition 5 holds and hence the theorem is proved.

Example 5. Let $\mathbf{L}_{3}=\left\langle\{1,2,3\}, \circ, 1,{ }^{-1}\right\rangle$ be an LA-polygroup with the Cayley (Table 6).

Then, $\mathbf{M}_{3}=\mathbf{L}_{3}\left\{\{4,5\}^{\diamond}\right.$ is an LA-polygroup with five elements and the Cayley (Table 7), where $M_{3}=\{1,2,3,4,5\}$.

This is an LA-polygroup and not a polygroup.

\section{Cayley Graphs over LA-Groups and LA- Polygroups}

Definition 6. Suppose that $\mathbf{G}$ is an LA-group and $C$ is a subset of $G$ such that 
TABLE 6: LA-polygroup.

\begin{tabular}{cccc}
\hline$\circ$ & 1 & 2 & 3 \\
\hline 1 & 1 & 2 & 3 \\
2 & 3 & $\{2,3\}$ & $\{1,2,3\}$ \\
3 & 2 & $\{1,2,3\}$ & $\{2,3\}$ \\
\hline
\end{tabular}

TABLE 7: LA-polygroup.

\begin{tabular}{cccccc}
\hline$\uplus$ & 1 & 2 & 3 & 4 & 5 \\
\hline 1 & 1 & 2 & 3 & 4 & 5 \\
2 & 3 & $\{2,3\}$ & $\{1,2,3\}$ & 4 & 5 \\
3 & 2 & $\{1,2,3\}$ & $\{2,3\}$ & 4 & 5 \\
4 & 4 & 4 & 4 & $\{1,2,3\}$ & 5 \\
5 & 5 & 5 & 5 & 5 & $\{1,2,3,4\}$ \\
\hline
\end{tabular}

(i) $1 \notin C$,

(ii) $C^{-1}=C$; then, Cayley graph Cay $(\mathbf{L A G}, C)$ of $\mathbf{G}$ relative to $C$ is the simple graph which has vertex set $G$ and edge set

$D=\{\{g, g s\} \mid g \neq g s, \quad$ where $g \in G$ and $s \in C\}$.

Example 6. The Cayley graphs of the LA-group $\mathbf{G}_{5}=\left(\left\{t_{1}, t_{2}, t_{3}, t_{4}, t_{5}\right\}, *\right)$ given in Example 1, with connection sets $\left\{t_{2}\right\}$ and $\left\{t_{2}, t_{3}\right\}$, are shown in Figures 1 and 2 . For $C=\left\{t_{2}\right\}$, we have

$$
\begin{aligned}
\left\{t_{1}, t_{1} * t_{2}\right\} & =\left\{t_{1}, t_{2}\right\}\left\{t_{2}, t_{2} * t_{2}\right\} \\
& =\left\{t_{2}, t_{1}\right\}\left\{t_{3}, t_{3} * t_{2}\right\} \\
& =\left\{t_{3}, t_{5}\right\}\left\{t_{4}, t_{4} * t_{2}\right\} \\
& =\left\{t_{4}, t_{4}\right\}\left\{t_{5}, t_{5} * t_{2}\right\} \\
& =\left\{t_{5}, t_{3}\right\} .
\end{aligned}
$$

For $C=\left\{t_{2}, t_{3}\right\}$, we have

$$
\begin{aligned}
\left\{t_{1}, t_{1} * t_{2}\right\} & =\left\{t_{1}, t_{2}\right\}\left\{t_{3}, t_{3} * t_{2}\right\} \\
& =\left\{t_{3}, t_{5}\right\}\left\{t_{1}, t_{1} * t_{3}\right\} \\
& =\left\{t_{1}, t_{3}\right\}\left\{t_{4}, t_{4} * t_{3}\right\} \\
& =\left\{t_{4}, t_{5}\right\} .
\end{aligned}
$$

Definition 7. Given an LA-polygroup $\mathbf{L}=\left\langle L, \circ, e,,^{-I}\right\rangle$ and $L \subseteq C \neq \varnothing$ such that $\left(C=C^{-I}\right)$, say the connection set. Then, we define the generalized Cayley graph over LA-polygroup GCLAP $(\mathbf{L} ; C)$ which is the simple graph having vertex set $L$ and the edge set

$$
D=\{\{d, f\} \mid d \neq f \text { and }(d \circ e) \circ f \cap C \neq \varnothing\} .
$$

If we have an LA-polygroup $\mathbf{L}$ and a connection set $C$ such that GCLAP $(\mathbf{L} ; C) \cong \Lambda$, then the graph $\Lambda$ is known as a GCLAP-graph.

Here, we give few examples of GCLAP-graphs.

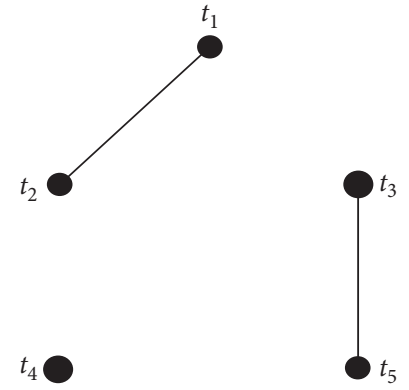

Figure 1: Cay $\left(\mathbf{L A G}_{5},\left\{t_{2}\right\}\right)$.

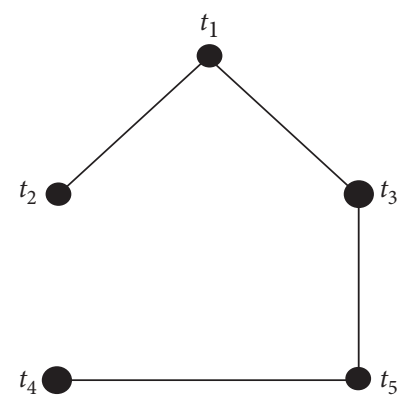

Figure 2: Cay $\left(\mathbf{L A G}_{5},\left\{t_{2}, t_{3}\right\}\right)$.

Example 7. The generalized Cayley graph of the left almost polygroup $\mathbf{L}_{4}=\left\langle\{1,2,3,4\}, \circ, 1,,^{-1}\right\rangle$, with connection set $C=\{3,4\}$ which is shown in Figure 3, where" " is defined in Table 8.

Example 8. The generalized Cayley graph of the left almost polygroup $\mathbf{L}_{5}=\left\langle\{1,2,3,4,5\}, \circ, 1,{ }^{-1}\right\rangle$ with connection set $\{2,3\}$ which is shown in Figure 4 , where" $\circ$ " is defined in Table 9.

\section{Which Simple Graphs Are GCLAP-Graphs?}

First, we point out a few types of simple graphs that are GCLAP-graphs. After that, we infer that each simple graph of order three, four, and five (except cycle graph of order five which may or may not be a GCLAP-graph) is a GCLAPgraph.

Lemma 1. Every Cayley graph is a GCLAP-graph.

Proof. Since every LA-group is an LA-polygroup, therefore, by Definition 7 , the result holds.

Lemma 2. Every complete graph of order at least three is a GCLAP-graph.

Proof. Let $\left\langle Q_{n}, *, t_{1}^{-1}\right\rangle$ be an LA-polygroup, where $n \geq 3$ (as defined in Example 2). Then, $\operatorname{GCLAP}\left(\mathbf{Q}_{n} ; Q_{n} \backslash\left\{t_{1}\right\}\right)$ are isomorphic to the complete graphs on $n$ vertices, where $n \geq 3$. Hence, it is proved. 


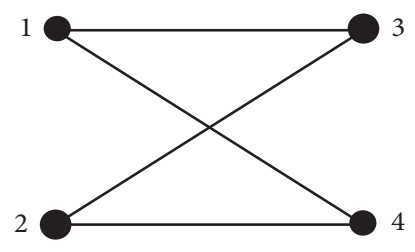

Figure 3: GCLAP $\left(\mathbf{L}_{4} ;\{3,4\}\right)$.

TABLE 8: LA-polygroup.

\begin{tabular}{ccccc}
\hline$\circ$ & 1 & 2 & 3 & 4 \\
\hline 1 & 1 & 2 & 3 & 4 \\
2 & 3 & $\{1,2,3\}$ & $\{2,3,4\}$ & 2 \\
3 & 2 & $\{2,3,4\}$ & $\{1,2,3\}$ & 3 \\
4 & 4 & 3 & 2 & 1 \\
\hline
\end{tabular}

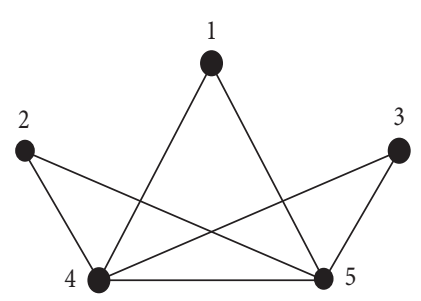

Figure 4: GCLAP $\left(\mathbf{L}_{5} ;\{4,5\}\right)$.

TABLE 9: LA-polygroup.

\begin{tabular}{cccccc}
\hline$\circ$ & 1 & 2 & 3 & 4 & 5 \\
\hline 1 & 1 & 2 & 3 & 4 & 5 \\
2 & 3 & $\{2,3\}$ & $\{1,2\}$ & 4 & 5 \\
3 & 2 & $\{1,3\}$ & $\{2,3\}$ & 4 & 5 \\
4 & 4 & 4 & 4 & $\{1,2,3,4,5\}$ & $\{4,5\}$ \\
5 & 5 & 5 & 5 & $\{4,5\}$ & $\{1,2,3,4,5\}$ \\
\hline
\end{tabular}

Lemma 3. Show that each star graph of order at least three is a GCLAP-graph.

Proof. Suppose that $\mathbf{L}=\mathbf{G}_{n}\left\{t_{n+1}\right\}^{\diamond}$, where $n \geq 3$ and $\mathbf{G}_{n}$ is defined in Example 1. Then, Cayley table for $\mathbf{L}$ is given in Table 10.

Now, by considering connection set $C=\left\{t_{n+1}\right\}$, we can see that $S_{n} \cong \operatorname{GCLAP}(\mathbf{L} ; C)$.

Lemma 4. If $\Phi$ is a GCLAP-graph. Then, show that $\Phi \cup n K_{1}$ is also a GCLAP-graph, where $n \geq 1$.

Proof. Let $\Phi$ be a GCLAP-graph. Then, we have a left almost polygroup $\mathbf{L}$ and a connection set $C$ such that $\Phi \cong \operatorname{GCLAP}(\mathbf{L} ; C)$. Suppose that $\Phi_{n}=\Phi \cup n K_{1}$ and $Z_{m}=\cup_{t=1}^{m}\left\{z_{t}\right\}$. By Extension (II) and Definition 7, we have $\Phi_{1} \cong \operatorname{GCLAP}\left(\mathbf{L}\left\{Z_{1}\right\}^{\diamond} ; C\right)$. Now, by using induction, $\Phi_{m} \cong \operatorname{GCLAP}(\mathbf{Q} ; C)$, where $\mathbf{Q}=\mathbf{L}\left\{Z_{m}\right\}^{\diamond}$ and $C$ is a connection set. Hence, $\Phi_{m+1} \cong \operatorname{GCLAP}\left(\mathbf{Q}\left\{z_{m+1}\right\}^{\diamond} ; C\right)$. Thus, $\Phi_{n}$ is a GCLAP - graph for every $n \in \mathbb{N}$.
TABLE 10: LA-polygroup.

\begin{tabular}{|c|c|c|c|c|c|c|c|}
\hline$\biguplus$ & $t_{1}$ & $t_{2}$ & . & . & . & $t_{n}$ & $t_{n+1}$ \\
\hline$t_{1}$ & & & & & & & $t_{n+1}$ \\
\hline$t_{2}$ & & & & & & & $t_{n+1}$ \\
\hline$\cdot$ & & & $\mathbf{G}_{n}$ & & & & $\cdot$ \\
\hline . & & & & & & & . \\
\hline . & & & & & & & . \\
\hline$t_{n}$ & & & & & & & $t_{n+1}$ \\
\hline$t_{n+1}$ & $t_{n+1}$ & $t_{n+1}$ & . & . & $\cdot$ & $t_{n+1}$ & $\left\{t_{1}, t_{2}, t_{3}, \ldots, t_{n}\right\}$ \\
\hline
\end{tabular}

Definition 8. Let $m \in\{1,2,3, \ldots\}$ and $q \in\{2,3,4, \ldots$, $m-1\}$. Then, the $(m, q)$-pseudo complete graph is known as the complement of the graph $S_{q} \cup N_{m-q}$ and represented by $\widehat{U}(m, q)$. A graph $\Phi$ is known as a pseudocomplete graph if $\Phi \cong \widehat{U}(m, q) \quad$ for some $m \in\{1,2,3, \ldots\}$ and $q \in\{2,3,4, \ldots, m-1\}$.

Example 9. Pseudocomplete graphs on five vertices are shown in Figure 5.

Lemma 5. If $m \in\{1,2,3, \ldots\}$ and $b, q \in\{2,3, \ldots, m-1\}$, then the following statements hold:

(i) $\widehat{U}(m, q)$ is connected,

(ii) If $q \neq b$ then $\widehat{U}(m, q) \neq \widehat{U}(m, b)$,

(iii) A connected graph $\Phi$ of order $m$ is a pseudocomplete graph $\Leftrightarrow$; it contains $K_{m-1}$ as a subgraph.

Lemma 6. All pseudocomplete graphs are GCLAP-graphs.

Proof. Let $\Phi=\widehat{U}(m, q)$ be a pseudocomplete graph, where $m \geq 1$ and $q \in\{2,3,4, \ldots, m-1\}$. Consider the LA-polygroup $\mathbf{L}=\mathbf{Q}_{3}\{\{4,5,6, \ldots, m\}\}$, where $\mathbf{Q}_{3}=\left\langle\{1,2,3\}, *, 1,{ }^{-1}\right\rangle$, given in Example 2, and the connection set $C=\{q+1, q+2, \ldots, m\}$. Then, $\overline{\operatorname{GCLAP}(\mathbf{L} ; C)}$ is isomorphic to the graph $S_{q} \cup N_{m-q}$. Hence, $\widehat{U}(m, q) \cong$ $\operatorname{GCLAP}(\mathbf{L} ; C)$.

Definition 9. The expansion of the graph $\Phi$, represented by $\Phi^{+}$, is the join of the graph $\Phi$ and $K_{1}$, i.e., $\Phi^{+}=\Phi \vee K_{1}$.

Lemma 7. Show that the expansion of a GCLAP-graph is a GCLAP-graph.

Proof. Let $\Lambda \cong \operatorname{GCLAP}(\mathbf{L} ; C)$, where $\mathbf{L}$ is a left almost polygroup having $n$ elements and $C$ is a connection set. Suppose that $\mathbf{Q}=\mathbf{L}\{\{n+1\}\}^{\diamond}$ and $C^{\prime}=C \cup\{n+1\}$. Then, $\Phi^{+} \cong \operatorname{GCLAP}\left(\mathbf{Q} ; C^{\prime}\right)$.

Definition 10. Let $\Phi=(G, D)$ be a graph, $g \in G$, and $f \notin G$. The faulty join graph, represented by $\nabla_{g}$, is the graph such that $\nabla_{g}(\Phi, f)=\left(G^{\prime}, D^{\prime}\right)$, where $G^{\prime}=G \cup\{f\} \quad$ and $D^{\prime}=D \cup\{\{f, h\}: g \neq h \in G\}$. Moreover, a graph is known as $\nabla_{1}$-graph if it is isomorphic to $\nabla_{1}(\Phi, f)$, where $\Phi=\operatorname{GCLAP}(\mathbf{L} ; C)$ such that $\mathbf{L}$ is a left almost polygroup having $n$ elements, $f \notin L$, and $C$ is a connection set. 

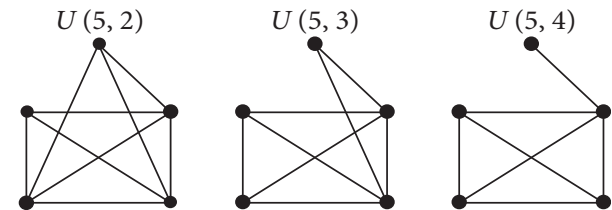

Figure 5: Pseudocomplete graphs on five vertices.

Lemma 8. Every $\nabla_{1}$-graph is a GCLAP-graph.

Proof. Suppose that $\Lambda$ is a $\nabla_{1}$-graph having $n$ vertices. So, we have a left almost polygroup $\mathbf{L}$ having $n$ elements and a connection set $C$ such that $\Lambda \cong \nabla_{1}(\operatorname{GCLAP}(\mathbf{L} ; C), n+1)$. Let $\mathbf{L}\{\{n+1\}\}=\mathbf{Q}$, then we have

$$
\nabla_{1}(\operatorname{GCLAP}(\mathbf{L} ; C), n+1) \cong \operatorname{GCLAP}(\mathbf{Q} ; C) .
$$

Hence, $\Lambda$ is a GCLAP-graph.

Up to here, we have determined a few types of GCLAPgraphs. Now, we confine ourselves to the graphs of order at most five (except cycle graph of order five) and show that every simple graph of order three, four, and five (except cycle graph of order five which may or may not be a GCLAPgraph) is a GCLAP-graph. In Appendix, we have shown all simple connected graphs of order three, four, and five (except cycle graph of order five) and denote them by $\Omega_{1}, \Omega_{2}, \ldots, \Omega_{28}$.

Theorem 3. All simple graphs of order three, four, and five (except cycle graph of order five which may or may not be a GCLAP-graph) are GCLAP-graphs.

Proof. Suppose that $\Phi$ is a simple graph of order three, four, and five (except cycle graph of order five). Then, as per the connectivity of $\Phi$, two cases can be thought of:

Case 1. If $\Phi$ is a connected graph, then we have six subcases:

Subcase (i) (cycles and complete graphs):

Consider the LA-polygroup $\mathbf{L}_{4}$ as in Example 7. Then, $\operatorname{GCLAP}\left(\mathbf{L}_{4} ;\{3,4\}\right) \cong \Omega_{5}$, so $\Omega_{5}$ is a GCLAP-graph. Also, since $\Omega_{2}, \Omega_{8}$, and $\Omega_{28}$ are complete graphs, therefore, by Lemma 2, they are GCLAP-graphs.

Subcase (ii) (star graphs): Think about LA-polygroup $\mathbf{L}_{3}$ as given in Example 5 , then $\operatorname{GCLAP}\left(\mathbf{L}_{3} ;\{2\}\right) \cong \Omega_{1}$. Also, since $\Omega_{4}$ and $\Omega_{9}$ are star graphs, so by Lemma 3, they are GCLAP-graphs.

Subcase (iii) (path graphs): Consider the LA-polygroup $\mathbf{L}_{4}$ and LA-group $\mathbf{G}_{5}$ as defined in Examples 7 and 6 , respectively. Then, $\Omega_{3} \cong \operatorname{GCLAP}\left(\mathbf{L}_{4} ;\{2\}\right)$, so $\Omega_{3}$ is a GCLAP-graph. Also, since Cay $\left(\mathbf{L A G}_{5},\left\{t_{2}, t_{3}\right\}\right) \cong \Omega_{11}$, therefore, by Lemma 1 , $\Omega_{11}$ is a GCLAP - graph.

Subcase (iv) (pseudocompleted graphs): Definition 8 implies that $\Omega_{6}, \Omega_{7}, \Omega_{22}, \Omega_{25}$, and $\Omega_{27}$ are pseudocomplete graphs and by Lemma 6 , they are GCLAP - graphs.
TABLE 11: LA-polygroup.

\begin{tabular}{ccccc}
\hline$\circ$ & 1 & 2 & 3 & 4 \\
\hline 1 & 1 & 2 & 3 & 4 \\
2 & 3 & $\{2,3,4\}$ & $\{1,2,4\}$ & $\{2,3\}$ \\
3 & 2 & $\{1,3,4\}$ & $\{2,3,4\}$ & $\{2,3\}$ \\
4 & 4 & $\{2,3\}$ & $\{2,3\}$ & $\{1,4\}$ \\
\hline
\end{tabular}

Subcase (v) (expansion graphs): Think about the LApolygroup $\mathbf{L}_{4}$ as in Example 7 and $\mathbf{L}_{6}=\left\langle\{1,2,3,4\}, \circ, 1,{ }^{-1}\right\rangle$, given in Table 11 .

$$
\begin{aligned}
& \Omega_{12} \cong\left(\operatorname{GCLAP}\left(\mathbf{L}_{4} ;\{4\}\right)\right)^{+}, \\
& \Omega_{16} \cong\left(\Omega_{1} \cup K_{1}\right)^{+}, \\
& \Omega_{17} \cong\left(\operatorname{GCLAP}\left(\mathbf{L}_{6} ;\{4\}\right)\right)^{+}, \\
& \Omega_{21} \cong \Omega_{4}^{+}, \\
& \Omega_{23} \cong \Omega_{3}^{+}, \\
& \Omega_{26} \cong \Omega_{5}^{+} .
\end{aligned}
$$

Definition 9 and above cases suggest that the graphs $\Omega_{12}, \Omega_{16}, \Omega_{17}, \Omega_{21}, \Omega_{23}$, and $\Omega_{26}$ are expansion graphs of GCLAP-graphs and by Lemma 7, we infer that they are GCLAP-graphs.

Subcase (vi) $\left(\nabla_{1} 0\right.$ graphs):

Consider three LA-polygroups $\mathbf{L}_{4}, \mathbf{L}_{2}$, and $\mathbf{L}_{1}$ as in Example 7, Example 4, and Example 3, respectively, then the following isomorphisms hold:

$$
\begin{aligned}
& \Omega_{10} \cong \nabla_{1}\left(\operatorname{GCLAP}\left(\mathbf{L}_{4} ;\{4\}\right), 5\right), \\
& \Omega_{13} \cong \nabla_{1}\left(\operatorname{GCLAP}\left(\mathbf{L}_{2} ;\{2\}\right), 5\right), \\
& \Omega_{14} \cong \nabla_{1}\left(\operatorname{GCLAP}\left(\mathbf{L}_{1} ;\{3\}\right), 5\right), \\
& \Omega_{18} \cong \nabla_{1}\left(\operatorname{GCLAP}\left(\mathbf{L}_{4} ;\{2\}\right), 5\right), \\
& \Omega_{24} \cong \nabla_{1}\left(\operatorname{GCLAP}\left(\mathbf{L}_{4} ;\{2,4\}\right), 5\right) .
\end{aligned}
$$

Thus, the graphs $\Omega_{10}, \Omega_{13}, \Omega_{14}, \Omega_{18}$, and $\Omega_{24}$ are $\nabla_{1}$-graphs. By Lemma 8 , we conclude that they are GCLAP-graphs. In the end, if we consider the left almost group $\mathbf{G}_{5}$ given in Example 6 and LA-polygroup, $\mathbf{L}_{7}=\left\langle\{1,2,3,4,5\}, \circ, 1,{ }^{-1}\right\rangle$ with the Cayley (Table 12).

Then, by Lemma 1 , we conclude that $\Omega_{19} \cong \operatorname{Cay}\left(\mathbf{L A G}_{5} ;\left\{a_{2}, a_{3}, a_{5}\right\}\right)$ and hence the following isomorphisms hold:

$$
\begin{aligned}
& \Omega_{15} \cong \operatorname{GCLAP}\left(\mathbf{L}_{7} ;\{3,5\}\right), \\
& \Omega_{20} \cong \operatorname{GCLAP}\left(\mathbf{L}_{7} ;\{3,4\}\right) .
\end{aligned}
$$

Thus, all simple connected graphs of order three, four, and five (except cycle graph of order five which may or may not be a GCLAP-graph) are GCLAP-graphs.

Case 2. If $\Phi$ is not connected. We have two subcases: Subcase (i): if $\Phi$ contains two nontrivial parts, then $\Phi$ is isomorphic to $K_{2} \cup K_{2}, K_{2} \cup \Omega_{1}$, and $K_{2} \cup \Omega_{2}$. We have $K_{2} \cup K_{2} \cong \operatorname{GCLAP}\left(\mathbf{L}_{1} ;\{3\}\right), \quad K_{2} \cup \Omega_{1} \cong \operatorname{GCLAP}\left(\mathbf{L}_{7} ;\right.$ 
TABLE 12: LA-polygroup.

\begin{tabular}{lllccc}
\hline$\circ$ & 1 & 2 & 3 & 4 & 5 \\
\hline 1 & 1 & 2 & 3 & 4 & 4 \\
2 & 2 & 1 & 3 & 5 & 5 \\
3 & 4 & 4 & $\{1,2\}$ & $3,2\}$ & 3 \\
4 & 3 & 3 & 5 & 3 \\
5 & 5 & 5 & 4 & $\{1,2\}$ \\
\hline
\end{tabular}
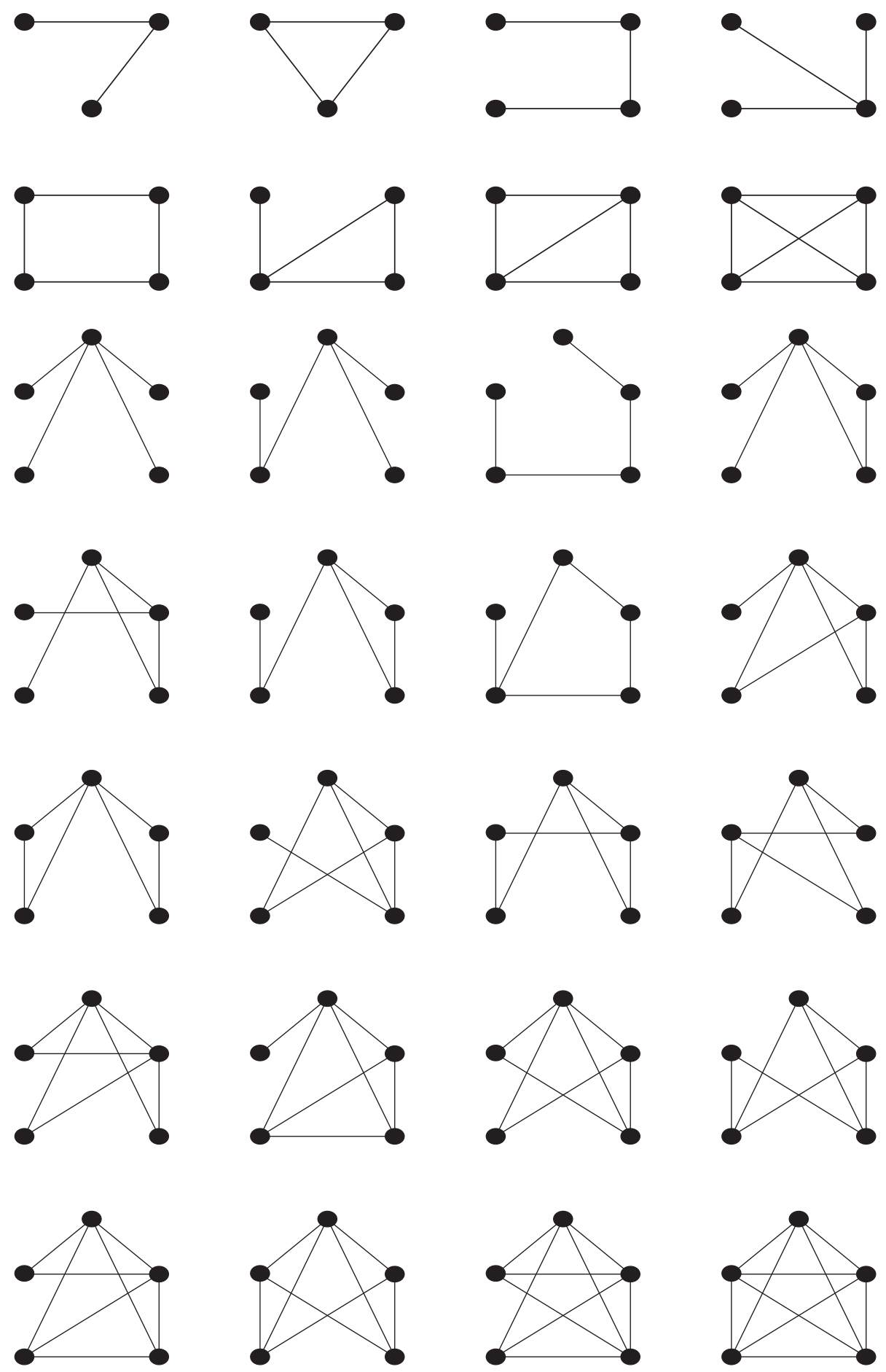

Figure 6: Connected simple graphs $\Omega_{1}, \Omega_{2}, \Omega_{3}, \ldots, \Omega_{28}$. 
$\{3\})$, and $K_{2} \cup \Omega_{2} \cong \operatorname{GCLAP}\left(\mathbf{L}_{7} ;\{2,5\}\right)$, where $\mathbf{L}_{1}$ and $\mathbf{L}_{7}$ are given in Example 3 and Subcase (vi), respectively. Subcase (ii): if $K_{1}$ is a subgraph of $\Phi$, then $\Phi \cong n K_{1} \cup \Lambda$, for $n \geq 1$ and $\Lambda$ is isomorphic to $K_{2} \cup K_{2}$ or isomorphic to $\mathrm{Cay}\left(\mathbf{L A G}_{3},\left\{t_{2}\right\}\right)$ or a connected graph of order $<5$. By Lemma 4, we conclude that $\Phi$ is a GCLAP - graph which completes the proof.

The question that usually comes to mind is what happens if $|\Phi|>5$; we present this query as an open problem $[28,29]$.

\section{Appendix}

There are 28 connected simple graphs of order three, four, and five (except cycle graph of order five) and they are denoted by $\Omega_{1}, \Omega_{2}, \Omega_{3}, \ldots, \Omega_{28}$ as given in Figure 6 .

\section{Data Availability}

No data were used to support this study.

\section{Conflicts of Interest}

The authors of this paper declare that they have no conflicts of interest.

\section{Acknowledgments}

This research was funded by the Deanship of Scientific Research at Princess Nourah Bint Abdulrahman University through the Fast-Track Research Funding Program.

\section{References}

[1] N. Biggs, E. K. Lloyd, and R. J. Wilson, Graph Theory, 17361936, Oxford University Press, Oxford, UK, 1986.

[2] A. Cayley, "On the theory of groups," Proceedings of the London Mathematical Society, vol. 9, pp. 126-233, 1878.

[3] P. S. Loh and L. J. Schulman, "Improved expansion of random cayley graphs," Discrete Mathematics and Theoretical Computer Science, vol. 6, pp. 523-528, 2004.

[4] A. Lubotzky, "Cayley graphs: eigenvalues, expanders and random walks," in Surveys in Combinatorics, 1995, pp. 155190, Cambridge University Press, Cambridge, UK, 1995.

[5] J. Morris, "Connectivity of Cayley graphs: a special family," Journal of Combinatorial Mathematics and Combinatorial Computing, vol. 20, pp. 111-120, 1996.

[6] M. H. Shahzamanian, M. Shirmohammadi, and B. Davvaz, "Roughness in Cayley graphs," Information Sciences, vol. 180, no. 17, pp. 3362-3372, 2010.

[7] F. Marty, "Sur une generalization de la notion de groupe," in Proceedings of the 8th Congress des Mathematiciens Scandinaves, pp. 45-49, Stockholm, Sweden, 1934.

[8] P. Corsini and V. Leoreanu, Applications of Hyperstructure Theory, Springer Science \& Business Media, Berlin, Germany, 2013.

[9] B. Davvaz, Semihypergroup Theory, Academic Press, Cambridge, MA, USA, 2016.

[10] P. Corsini, Prolegomena of Hypergroup Theory, Aviani editore, Meerut, India, 1993.

[11] B. Davvaz, Hyperring Theory and Applications, Bijan Davvaz Publications, Yazd, Iran, 2007.
[12] B. Davvaz, Polygroup Theory and Related Systems, World Scientific Publishing Co Pte Ltd., Hackensack, NJ, USA, 2013.

[13] T. Vougiouklis, Hyperstructures and their representations, Hadronic Press. Inc., Palm Harbor, FL, USA, 1994.

[14] S. Ioulidis, "Polygroups et certains de leurs properietes," Bulletin of the Malaysian Mathematical Sciences Society, vol. 39, no. 2, pp. 707-721, 1981.

[15] S. M. Anvariyeh, S. Mirvakili, and B. Davvaz, "Combinatorial aspects of n-ary polygroups and n-ary color schemes," $\mathrm{Eu}$ ropean Journal of Combinatorics, vol. 34, no. 2, pp. 207-216, 2013.

[16] S. D. Comer, "Extension of polygroups by polygroups and their representations using color schemes," in Universal Algebra and Lattice Theory, pp. 91-103, Springer, Berlin, Germany, 1983.

[17] S. D. Comer, "Polygroups derived from cogroups," Journal of Algebra, vol. 89, no. 2, pp. 397-405, 1984.

[18] C. Berge and C. Berge, Graphes et hypergraphes. 1970, Dunod, Paris, France, 1967.

[19] M. A. Kazım and M. Naseeruddin, "On almost semigroups," The Align Bulletin Mathematics, vol. 2, pp. 1-7, 1972.

[20] Q. Mushtaq and M. S. Kamran, "On left almost groups," Proceedings-Pakistan Academy of Sciences, vol. 33, pp. 53-56, 1996.

[21] K. Hila and J. Dine, "On hyperideals in left almost semihypergroups," ISRN Algebra, vol. 2011, Article ID 953124, 8 pages, 2011.

[22] N. Yaqoob, P. Corsini, and F. Yousafzai, "On intra-regular left almost semihypergroups with pure left identity," Journal of Mathematics, vol. 2013, Article ID 510790, 10 pages, 2013.

[23] V. Amjad, K. Hila, and F. Yousafzai, "Generalized hyperideals in locally associative left almost semihypergroups," New York Journal of Mathematics, vol. 20, 2014.

[24] N. Yaqoob, I. Cristea, M. Gulistan, and S. Nawaz, "Left almost polygroups," Italian Journal of Pure and Applied Mathematics, vol. 39, pp. 465-474, 2018.

[25] D. Heidari, M. Amooshahi, and B. Davvaz, "Generalized Cayley graphs over polygroups," Communications in Algebra, vol. 47, no. 5, pp. 2209-2219, 2019.

[26] J. A. Bondy and U. S. R. Murty, Graph Theory with Applications, Vol. 290, Macmillan, London, UK, 1976.

[27] V. I. Voloshin, Introduction to Graph and Hypergraph Theory, Nova Science Publishers, Hauppauge, New York, USA, 2009.

[28] D. J. Robinson, A Course in the Theory of Groups, Springer Science \& Business Media, Berlin, Germany, 2012.

[29] G. Smith and O. Tabachnikova, Topics in Group Theory, Springer Science \& Business Media, Berlin, Germany, 2000. 